\title{
Assessment of Ride Quality at Lane Change Maneuver Using Virtual Human Driver Model
}

\author{
Manyong Han ${ }^{1}$ Hyung Yun Choi $^{2}$ Akinari Hirao $^{3}$ Stefan Kirschbichler ${ }^{4}$ \\ ${ }^{1}$ Department of Mechanical Engineering, HongIk University, Korea, myhan@mail.hongik.ac.kr \\ ${ }^{2}$ Mechanical System Design Engineering Department, HongIk University, Korea, hychoi@hongik.ac.kr \\ ${ }^{3}$ Nissan Motor Co., Ltd., Japan, a-hirao@mail.nissan.co.jp \\ ${ }^{4}$ VIRTUAL VEHICLE, Austria, Stefan.Kirschbichler@v2c2.at
}

\begin{abstract}
The occupant kinematics occurring at a lane change maneuver affects the local ride quality. The precise analysis of the occupant kinematics requires a comprehensive understanding of the physiologic response to human body as well as the vehicle kinematics. A series of vehicle-based tests also confirmed that the alertness level of vehicle occupants is one of the important biomechanical elements. Therefore, it is necessary to have a virtual human body model (HBM), an occupant surrogate at CAE design process, with active muscle forces to represent the reflexive response of human beings. An active human body model that produces joint torques with PID closedloop control as mimicking a bracing action to keep the sitting posture against the external jerk has been developed. In this study, this active human body model is validated against the subject test by simulating the similar occupant kinematics at a single lane change maneuvers. To further verify the use of active HBM as a design tool, an artificial lane change maneuver with a reduced lateral jerk is fabricated and good matching occupant kinematics are predicted.
\end{abstract}

Keywords: $\quad$ Occupant kinematics, Ride quality, Lane change, Active human body model

\section{Introduction}

The demand of objective and quantitative tools for an assessment of the ride quality design of vehicle occupant is quite high. Since the principal subject of vehicle driving tends to migrate from human to machine as the autonomous vehicles prevail more in the fleet, the ride quality of passengers affected by the vehicle maneuvers moves from the driving skill of individual human to the engineering car design sector. Short-range maneuvers such as a lane change cause a lateral force on the vehicle that perturbs sitting occupants and arouse them to brace to maintain the equilibrium posture. This unavoidable muscular activity of vehicle occupants lead to the discomfort of riding, the poor ride quality.

In physics, jerk is the rate of change of acceleration, which is the derivative of acceleration with respect to time. The jerk has physiological effects on occupant's perception in a moving vehicle. Occupant tends to brace as maintaining a balanced sitting posture against external perturbations, the vehicle movements. When the changes of the vehicle movements are abrupt, the acting forces on the occupant become fast as well. Then, there would not be enough time for establishing a control loop to achieve this equilibrium by adjusting agonistic and antagonistic muscle tensions, i.e., beyond the physiological bounds. Therefore an excessive acceleration (force) and jerk (rate of force) in vehicle movement may result in an uncomfortable ride.

The ride quality of a vehicle maneuvering such as lane change can be assessed common by two physical quantities, the maximum acceleration and the maximum jerk which are effective to predict the local discomforts of occupants. Local discomfort typically arises when the acceleration reaches $2.45 \mathrm{~m} / \mathrm{s}^{2}$ (Schofield, 2001) or when the jerk reaches $0.9 \mathrm{~m} / \mathrm{s}^{3}$ (AASHTO, 2001). The average ride quality can be determined by using the standard ISO 2631 or the UIC ride quality note (Lauriks, 2003) which evaluates the acceleration data over time.

Takahashi et al (Takahashi, 2013) presented "Preview G-Vectoring Control" (PGVC), which is based on the "G-Vectoring Control" (GVC) scheme by MAZDA. In GVC, the longitudinal-acceleration control algorithm is based on the actual lateral jerk. PGVC decelerates a vehicle before it enters a curve, and is based on a new longitudinal-acceleration control algorithm which uses 
predicted and actual lateral jerk. They concluded that it was confirmed that PGVC could reduce the jerk without an excessive slow-down. And this could be a useful driver assistance system to reduce the driver's braking task.

The virtual human body model (HBM) has extensive applications in crashworthiness design of the vehicle to predict kinematics and injury risks of both occupant and pedestrian (www.GHBMC.com; EuroNCAP TB 024, 2017). Considering the upcoming trends in the automotive industry, namely active safety and autonomous driving, the rising demand of active human body model was brought up by Kleinbach and Fehr (Kleinbach, 2017). As the focus of vehicle safety is shifting towards scenarios with lower acceleration levels, traditional surrogate models including HBM developed for high acceleration levels no longer suffice. Authors presented an efficient way to model muscle forces of vehicle occupants as they maintain the postural stability during the ride. The active joint torque controlled by a proportional integral derivative (PID) closed loop was introduced at the elbow joint to simulate the voluntary and reflexive response of the human subjects. (Han, 2016) We further extended this active human body modeling scheme to the whole body to simulate vibration response to the uncoupled translational excitations (Choi, 2017).

Yamada et al (Yamada, 2016) used THUMS v5 model for simulating the occupant kinematics in the single lane change maneuvers. When the skeletal muscles were activated at the THUMS model, a less lateral displacement was found than in the case without muscle activation. That correlated well with the findings from their reference test (Kirschbichler, 2014).
In this study, a part of the outcomes from the vehiclebased tests at OM4IS (Occupant Model for Integrated Safety) project (Kirschbichler, 2014) is utilized to verify and validate the active HBM. There were two vehicle maneuvers, emergency braking, and single lane change. Also two kinds of seat conditions, series A: wood seat and lap belt and series B: cushioned seat and lap and shoulder belt. Among two test maneuvers, the single lane change (c.f., the other one was emergency braking) is selected for this study. The single lane change was conducted at $50 \mathrm{~km} / \mathrm{h}$ vehicle speed for 2 seconds period of time that produced a $10 \mathrm{~m} / \mathrm{s}^{2}$ peak lateral acceleration. This steering action was rather a swift movement like an evasive lane change to avoid a collision into the obstacle. The series A seat condition was selected because it provides simpler boundary condition at model simulation than series B.

\section{Analysis of the test data}

To investigate the human response to low-load situations prevalent in the pre-crash phase, a series of lane change tests had been conducted at OM4IS project (Kirschbichler, 2014). Twenty-one male test subjects (age: $33.4 \pm 8.8 \mathrm{y}$, mass: $78.5 \pm 6.3 \mathrm{~kg}$, height: $179.2 \pm 4.6$ $\mathrm{cm}$, sitting height: $91.0 \pm 2.0 \mathrm{~cm}$ ) were recruited as front seat passengers. They were requested to keep three contrasting awareness states. An unaware, an anticipated and an informed condition, respectively at each maneuver, which have different levels of active muscle contribution to the body kinematics during the lane change maneuvers. Table 1 shows age and body size of the test subjects actually participated the test. The number of subjects was reduced from 4 to 5 for the data analysis due to either missing or noisy signals.

Table 1. Age and Body Size of Test Subjects

\begin{tabular}{|c|c|c|c|c|}
\hline Awareness & $\begin{array}{c}\text { Number of } \\
\text { test subjects }\end{array}$ & $\begin{array}{c}\text { Age } \\
\text { (years old })\end{array}$ & $\begin{array}{c}\text { Weight } \\
(\mathrm{kg})\end{array}$ & $\begin{array}{c}\text { Height } \\
(\mathrm{cm})\end{array}$ \\
\hline Unawared & $22\left(18^{*}\right)$ & $32.2 \pm 8.6$ & $77.4 \pm 6.7$ & $179.4 \pm 4.3$ \\
\hline Anticipated & $20\left(16^{*}\right)$ & $31.5 \pm 8.3$ & $76.9 \pm 6.7$ & $179.2 \pm 4.2$ \\
\hline Informed & $21\left(17^{*}\right)$ & $32.2 \pm 8.7$ & $76.9 \pm 6.5$ & $179.1 \pm 4.1$ \\
\hline
\end{tabular}

*: number of test subjects used for data analysis

Table 2. Mean $\left( \pm \sigma^{*}\right)$ peak and maximum values at lane change maneuvers

\begin{tabular}{|c|c|c|c|c|c|c|}
\hline \multirow{3}{*}{ Awareness } & \multicolumn{2}{|c|}{ Vehicle peak } & \multicolumn{2}{c|}{ Head maximum } & \multicolumn{2}{c|}{ Torso maximum } \\
\cline { 2 - 7 } & $\begin{array}{c}\text { lateral acc. } \\
\left(\mathrm{m} / \mathrm{s}^{2}\right), A_{y}\end{array}$ & $\begin{array}{c}\text { lateral jerk } \\
\left(\mathrm{m} / \mathrm{s}^{3}\right), J_{y}\end{array}$ & $\begin{array}{c}\text { lateral disp. } \\
(\mathrm{mm}), D_{h y}\end{array}$ & $\begin{array}{c}\text { rolling angle } \\
(\text { degree }), R_{h x}\end{array}$ & $\begin{array}{c}\text { lateral disp. } \\
(\mathrm{mm}), D_{t y}\end{array}$ & $\begin{array}{c}\text { rolling angle } \\
(\text { degree }), R_{t x}\end{array}$ \\
\hline Unaware & $9.9( \pm 0.2)$ & $41.2( \pm 3.7)$ & $271.7( \pm 96.6)$ & $10.6( \pm 10.7)$ & $216.1( \pm 63.8)$ & $14.9( \pm 7.3)$ \\
\hline Anticipated & $9.9( \pm 0.4)$ & $39.6( \pm 5.1)$ & $251.3( \pm 107.0)$ & $13.3( \pm 11.5)$ & $189.6( \pm 67.7)$ & $14.5( \pm 7.0)$ \\
\hline Informed & $9.9( \pm 0.4)$ & $40.2( \pm 4.5)$ & $193.1( \pm 84.6)$ & $9.9( \pm 8.5)$ & $147.0( \pm 58.5)$ & $9.2( \pm 4.8)$ \\
\hline
\end{tabular}


The averaged vehicle and occupant kinematics are shown in Figure 1. The first peak value of each curve involves the most meaningful physical quantity of the cause-and-effect relationship between the vehicle and occupant kinematics. Table 2 lists those mean peak vehicle and maximum head and torso values at lane change maneuvers. The more comprehensive kinematic data with standard deviations about all test subjects are presented in the references (Kirschbichler, 2014).

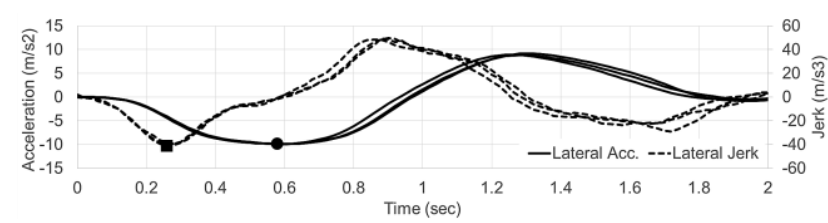

a) Lateral Acceleration $\left(\mathrm{A}_{\mathrm{y}}\right)$ and Jerk $\left(\mathrm{J}_{\mathrm{y}}\right)$ of Vehicle

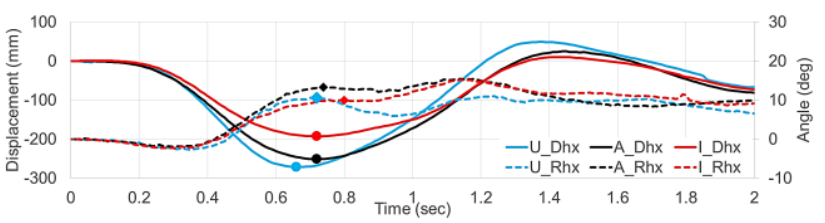

b) Lateral head displacement $\left(\mathrm{D}_{\mathrm{hy}}\right)$ and rolling angle $\left(\mathrm{R}_{\mathrm{hx}}\right)$ of test subject

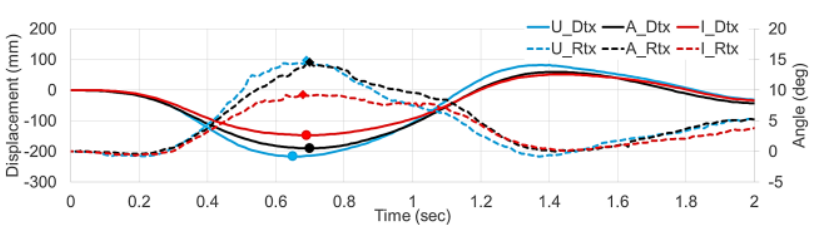

c) Lateral torso displacement $\left(D_{t y}\right)$ and rolling angle $\left(R_{t x}\right)$ of test subject

Figure 1. Mean profiles of vehicle and occupant kinematics at lane change maneuvers. Dot on each curve indicates peak (or maximum) value in Table 2 (U: Unaware, A: Anticipated, I: Informed)

\subsection{Statistical Analysis}

Data of each individual test subject are statistically analyzed. Even though the protocol of the lane change test was designed to provide the same vehicle motions for each lane change maneuver to identify and quantify the occupant kinematics with three awareness conditions, the peak vehicle lateral jerk has substantial variation. It reaches from $50.0 \mathrm{~m} / \mathrm{s}^{3}$ to $30.0 \mathrm{~m} / \mathrm{s}^{3}$ (mean: $40.0 \mathrm{~m} / \mathrm{s}^{3}$ and $\sigma: 4.4 \mathrm{~m} / \mathrm{s}^{3}$ ) while very small deviation of the peak lateral acceleration from $10.2 \mathrm{~m} / \mathrm{s}^{2}$ to $9.4 \mathrm{~m} / \mathrm{s}^{2}$ (mean: $9.9 \mathrm{~m} / \mathrm{s}^{2}$ and $\sigma: 0.3 \mathrm{~m} / \mathrm{s}^{2}$ ). The effect of peak lateral jerk on the maximum head lateral displacement was statistically analyzed but no significant correlation was found $(\mathrm{p}=0.214, \mathrm{R} 2=0.031$ ). (See Figure 2)

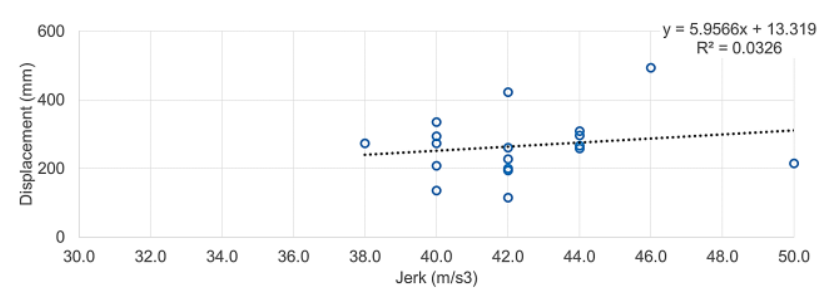

a) Unaware

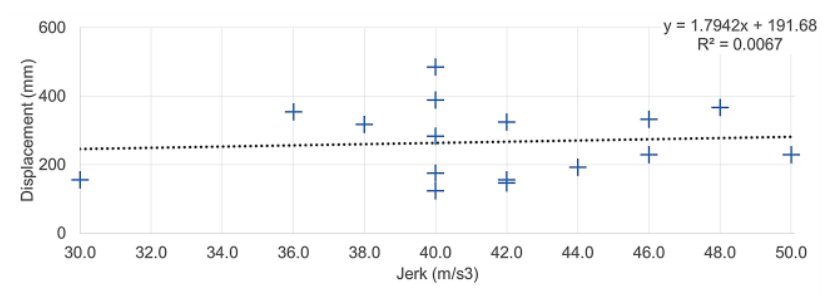

b) Anticipated

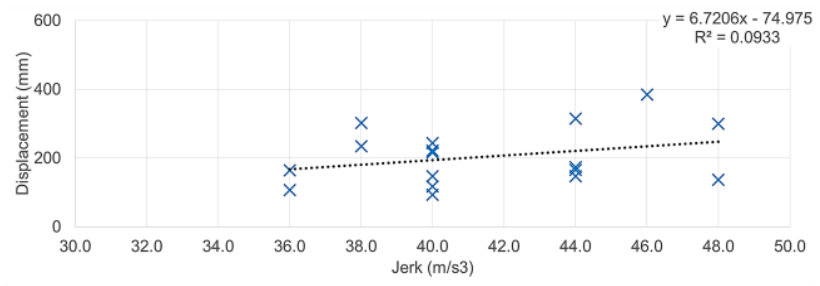

c) Informed

Figure 2. Relation between vehicle peak lateral jerk and maximum head lateral displacement of test subject

\section{Virtual Human Body Model}

The whole body human model developed for the ride comfort study (Choi, 2017) is adopted as a virtual occupant surrogate to simulate the occupant kinematics at lane change maneuver. This human body model has a multi-body system consisting of 15 body regions and 14 articulated joints. The skin morphology presents an average North American 50 ${ }^{\text {th }} \%$ tile male population (Kim, 2007; SizeUSA survey, 2000-03) which has 86kg and $178 \mathrm{~cm}$ of body size. As discussed in detail in section 2 , analysis of the test data, marker trajectories were used to estimate the position and orientation, the upper body kinematics (head and torso). For improving the computational efficiency, the modeling of upper and lower limbs are switched to non-deformable articulations and rigidly mounted to upper and lower trunks. The total number of body segments then become five, head, neck, upper trunk, center trunk, and lower trunk and four joints in the trunk as listed in Table 3. The hand position is also changed from the steering wheel to laps representing a passenger posture as shown in Figure 3. 


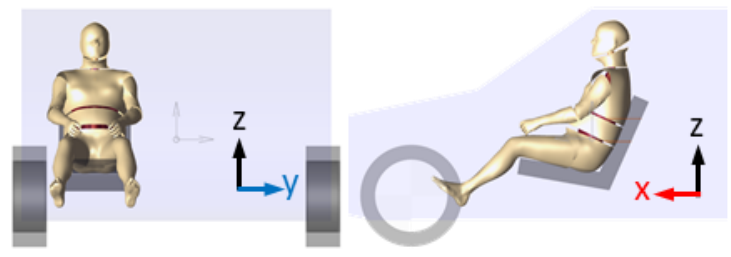

Figure 3. Human body model in multi-body system

Table 3. Four Articulated Joints with Their Anatomical Positions

\begin{tabular}{|c|c|c|c|}
\hline$\#$ & Articulated joint & DOF & $\begin{array}{c}\text { Anatomical } \\
\text { position }\end{array}$ \\
\hline 1 & Head-neck & 3 & OC joint \\
\hline 2 & Neck-Upper trunk & 3 & C7/T1 \\
\hline 3 & Upper-Center trunk & 3 & T12/L1 \\
\hline 4 & Center-Lower trunk & 3 & L5/S1 \\
\hline
\end{tabular}

Voluntary and reflexive muscle activation of a vehicle occupant is modeled by active joint elements at each anatomical joint position. There are two basic elements at each joint, i.e., the passive kinematic joint element and the torque actuator. Contrastively to voluntary activation of individual muscles, i.e., the pretension and consequent stiffening of the articulated joint represented by the passive kinematic joint element, a vestibular reflexive muscle activation for the posture stabilization is modeled by the introduction of active torques with PID closed-control (Choi, 2017). The active torque, the control signal, is a sum of proportional, integral, and derivative terms between the current and the reference (initial) joint angles. The gain values at the PID control determine the rates of torque generation. Faster torque generation with larger gain values stands for the pre-recognition of the upcoming external perturbation. Each term at PID control can be adjusted to calibrate the rate of muscle recruitment for fine control of the reflexive response of the human occupant. Authors of this paper showed a successful application of the proposing active joint modeling with the elbow reacting to the jerk loading (Choi, 2016; Han, 2016).

\section{Simulation of Single Lane Change}

\subsection{Validation of human body model against the single lane change test data.}

The forward $\left(\mathrm{V}_{\mathrm{x}}\right)$, lateral $\left(\mathrm{V}_{\mathrm{y}}\right)$, and yaw angular $\left(\mathrm{W}_{\mathrm{z}}\right)$ velocities of the test vehicle measured at single lane change maneuvers in Figure 4 was used as the kinematic input condition for the lane change simulation.

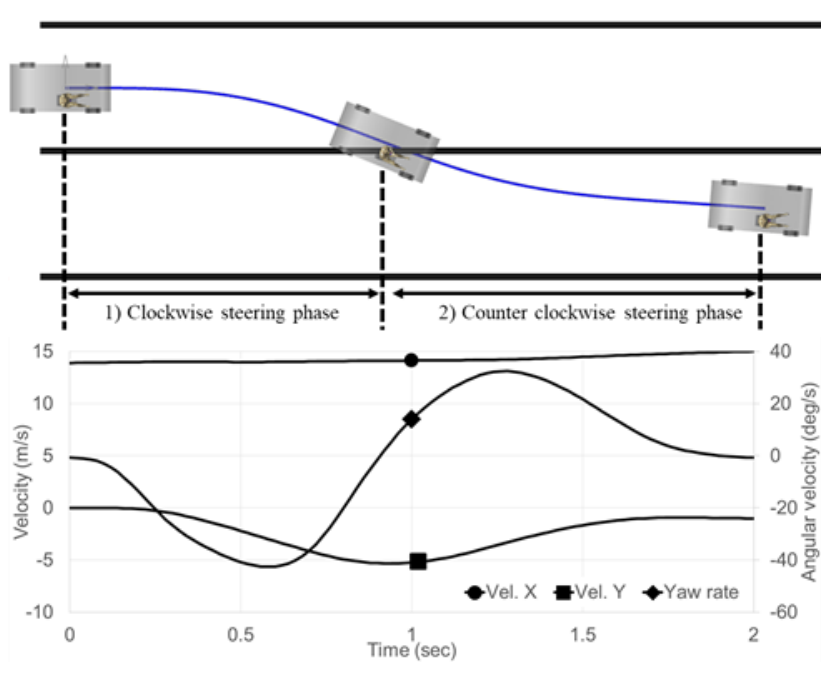

Figure 4. Kinematic input condition of single lane change maneuver for the simulation: 1) clockwise steering phase ( $\sim 0.95 \mathrm{~s}), 2)$ counter clockwise steering phase $(0.95 \sim 2.0 \mathrm{~s})$

Considering the lap belt restraining with a probable slackening, the lower trunk of HBM is tied to the seat cushion by an elastic spring element, which allows a small amount $(<20 \mathrm{~mm})$ of buttock sliding over the seat cushion. A sliding contact, the polygon-to-polygon contact type at SimulationX (ESI ITI) is defined between the seat back and occupant with 0.1 frictional coefficients.

A heuristic calibration of PID gains and neural delay time were carried out until the simulated outcomes, the time plot of the head and torso lateral displacements become analogous to the test measurement. In order to quantify the correlation between the test and simulation, the CORA (CORrelation and Analysis, http://www.pdborg.com/de/information/18-cora-download.html) score was adopted.. Figure 5 shows the comparison of head and torso lateral displacements $\left(D_{y}\right)$ between test and simulation for three awareness conditions. The PID gains and neural delay times, applied to the simulation are listed together with the CORA scores in Table 4. The neural delay times were estimated based on the onset muscle latency measured at the test. (Huber, 2013) The calculation of CORA score was divided into 2 sections, phase $1(0.0-0.95 \mathrm{sec}$, clockwise steering) and phase 2 (0.95-2.0sec. counter clockwise steering). In phase 1 a significant higher average CORA score was found than in phase 2, i.e., 0.94 versus 0.46 . In the PID closed-loop control, the joint torque was activated to minimize the current angles of each body segment tilting away from the initial vertical axis, i.e., upright alignment of headneck-torso. This is assuming that the test subject tries to keep the initial upright sitting posture. The criterion for a termination of heuristic calibration trial was reaching 0.8 of the CORA score for the phase $1(0.0-0.95 \mathrm{sec})$. 
Table 4. Calibrated PID gains, neural delay times and CORA scores

\begin{tabular}{|c|c|c|c|c|c|c|c|}
\hline \multirow{2}{*}{ Awareness } & \multicolumn{3}{|c|}{ PID Gains } & \multirow{2}{*}{$\begin{array}{c}\text { Neural } \\
\text { delay } \\
\text { time }(\mathrm{ms})\end{array}$} & \multicolumn{3}{|c|}{ CORA score* } \\
\hline & $\mathrm{K}_{\mathrm{P}}$ & $\mathrm{K}_{\mathrm{I}}$ & $\mathrm{K}_{\mathrm{D}}$ & & $\operatorname{Head}\left(D_{\text {hy }}\right)$ & Torso(D $\left.D_{\text {ty }}\right)$ & Total \\
\hline Unaware & 35 & 0.01 & 500 & 200 & $\begin{array}{c}0.879 \\
{[0.959,0.604]}\end{array}$ & $\begin{array}{c}0.738 \\
{[0.857,0.441]}\end{array}$ & $\begin{array}{c}0.809 \\
{[0.908,0.522]}\end{array}$ \\
\hline Anticipitated & 39 & 0.01 & 700 & 170 & $\begin{array}{c}0.815 \\
{[0.995,0.418]}\end{array}$ & $\begin{array}{c}0.776 \\
{[0.906,0.457]}\end{array}$ & $\begin{array}{c}0.795 \\
{[0.950,0.438]}\end{array}$ \\
\hline Informed & 55 & 0.01 & 2000 & 100 & $\begin{array}{c}0.646 \\
{[0.962,0.219]}\end{array}$ & $\begin{array}{c}0.842 \\
{[0.970,0.596]}\end{array}$ & $\begin{array}{c}0.744 \\
{[0.966,0.408]}\end{array}$ \\
\hline
\end{tabular}

*: calculated for total $(0.0-2.0 \mathrm{sec})$ [phase \#1(0.0-0.95sec), phase \#2(0.95-2.0sec)]

The simulated upper body motion of the unaware case is displayed in the Figure 6. The associated active torque generated at the articulated joint at the centerlower trunk (L5-S1) is also presented in Figure 7.

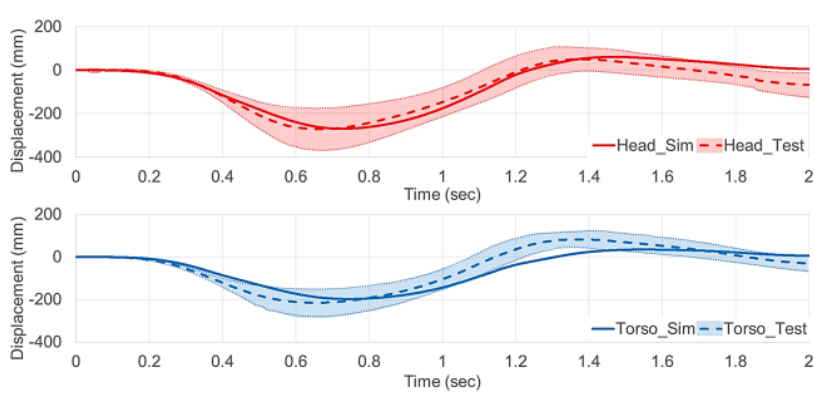

a) Unaware
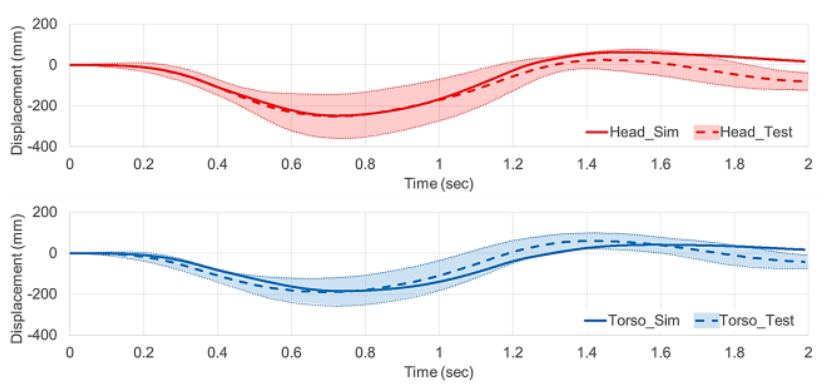

b) Anticipated

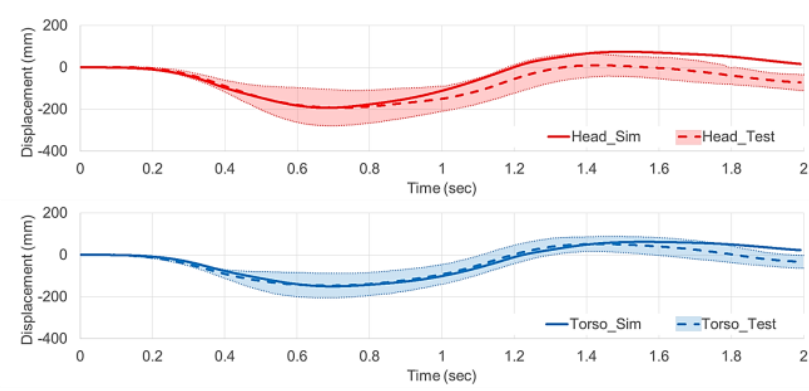

c) Informed

Figure 5. Comparison of head and torso lateral displacements $\left(\mathrm{D}_{\mathrm{y}}\right)$ between test and simulation
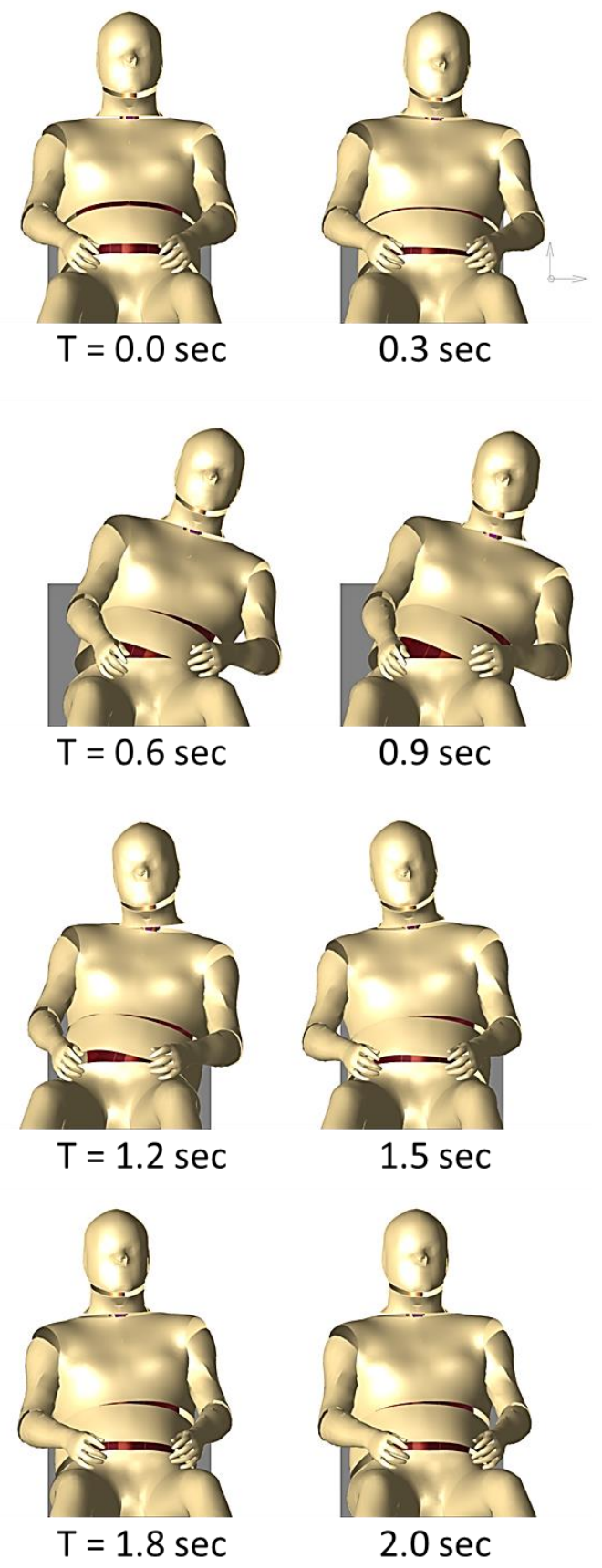

Figure 6. Frontal view of occupant kinematics at simulation (Unaware case). 


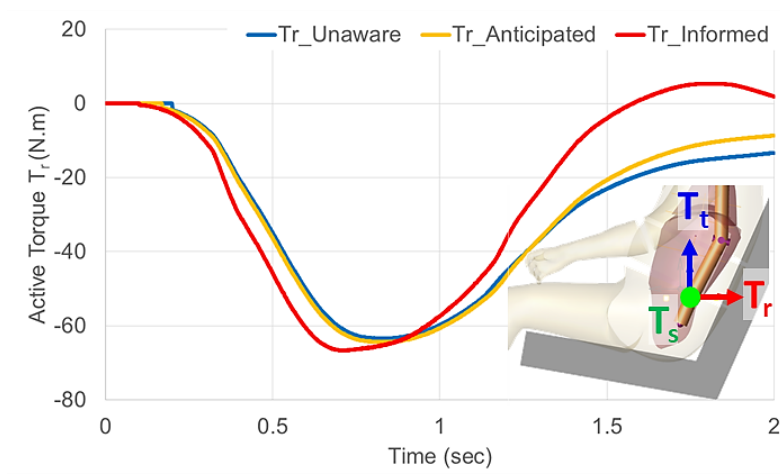

Figure 7. Simulated active torque generation at centerlower trunk joint r-direction (L5/S1 r-direction)

\subsection{Creation of a lane change maneuver with reduced lateral jerk}

An artificial driving movement for a single lane change is created to produce the same lateral displacement, but with a reduced maximum lateral jerk as seeking a smoother vehicle maneuver than at the human driving test in section 2. It is hypothesized that the test vehicle was driven to take an evasive lane change by a human driving expert just to avoid a crash but bearing no comfort ride in mind. However, a well-programmed machine, e.g., highly autonomous vehicle may steer the vehicle to take the evasive maneuver not just for a crash avoidance but also for a good ride quality of the passenger. Figure 8 shows the fabricated vehicle movement at a single lane change as compared with the measured one at the human driving test. The artificial maneuver was fabricated to have a similar shape of jerk time history as the original test but with more smooth profile as shown in Figure 8. In spite of the reduced maximum lateral jerk at the fabricated maneuver by $27.8 \%$ $\left(40.0 \mathrm{~m} / \mathrm{s}^{3} \rightarrow 28.9 \mathrm{~m} / \mathrm{s}^{3}\right)$, the lateral acceleration and displacement are kept almost same as those at the actual test (also see the Fig, 8).

The same active human body model used for the validation of the single lane change test in Section 4.1 is utilized to predict upper body kinematics at the created lane change maneuver with the reduced maximum lateral jerk. The predicted occupant kinematics at the created lane change maneuver with the reduced jerk revealed decreased lateral head and torso excursions by 5-6\% as expected. (see the Figure 9)

The joint work, the product of joint torque and the angle, can be regarded as a muscle energy produced to maintain a desired posture against the external perturbation, the lateral acceleration (or jerk) at the single lane change in this study. The simulated change of muscle energy at four articulated joints from the original test condition to the fabricated reduced jerk condition is shown in Figure 10, which is around $10 \%$ reduction in total for all three awareness conditions.

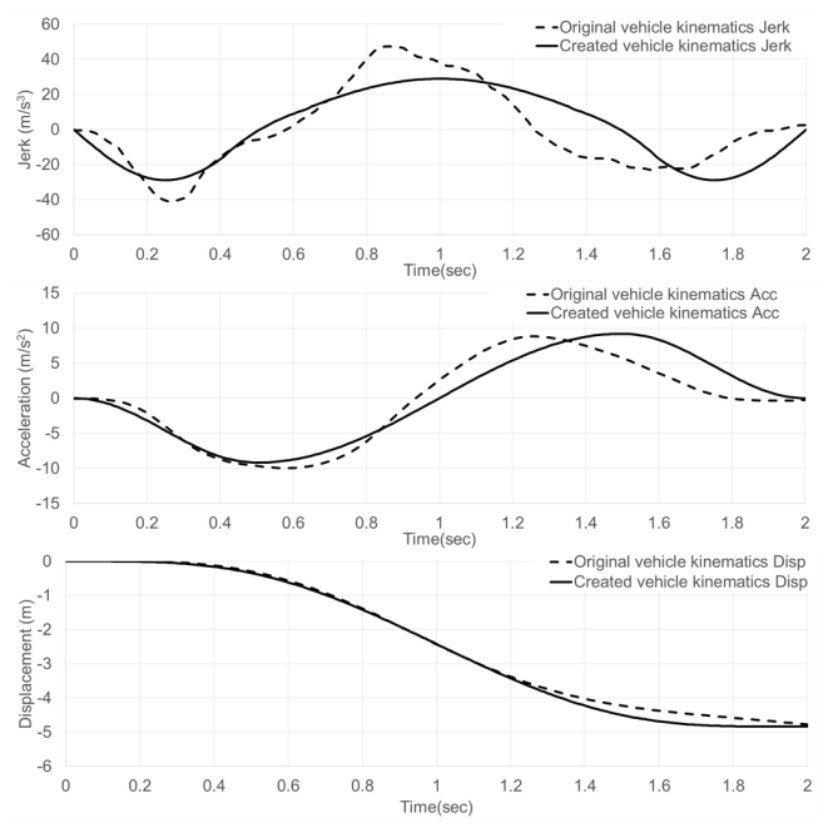

Figure 8. Comparison of vehicle kinematics between at the actual test and at the created maneuver with a reduced lateral jerk.

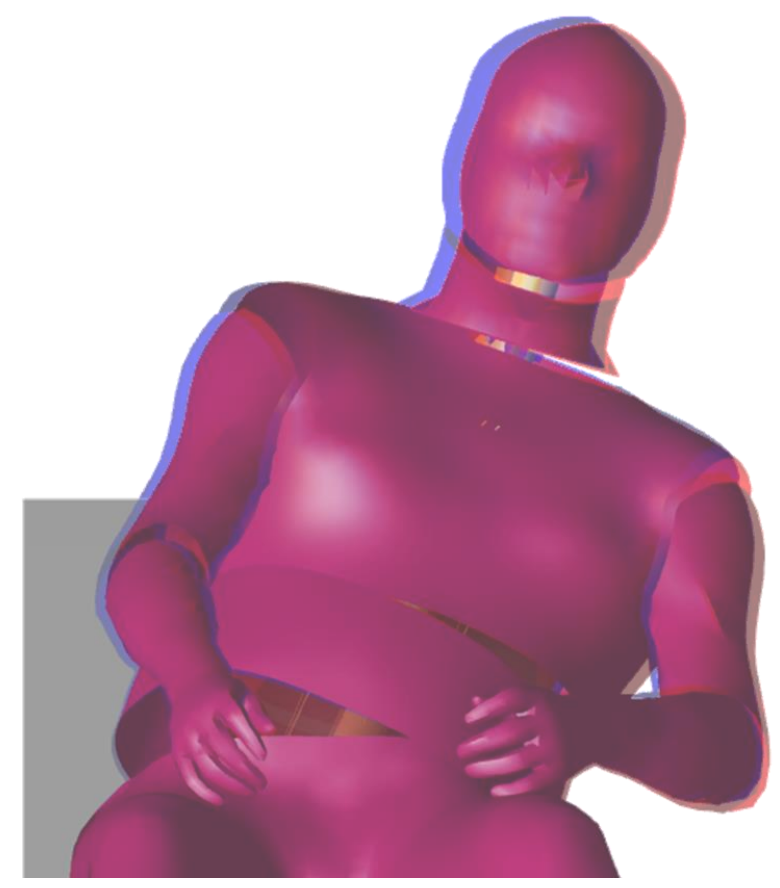

Figure9. Comparison of simulated maximum head excursions for unaware case between the original test condition (red, $0.727 \mathrm{sec}$ ) and the reduced jerk condition (blue, $0.695 \mathrm{sec}$ ) 


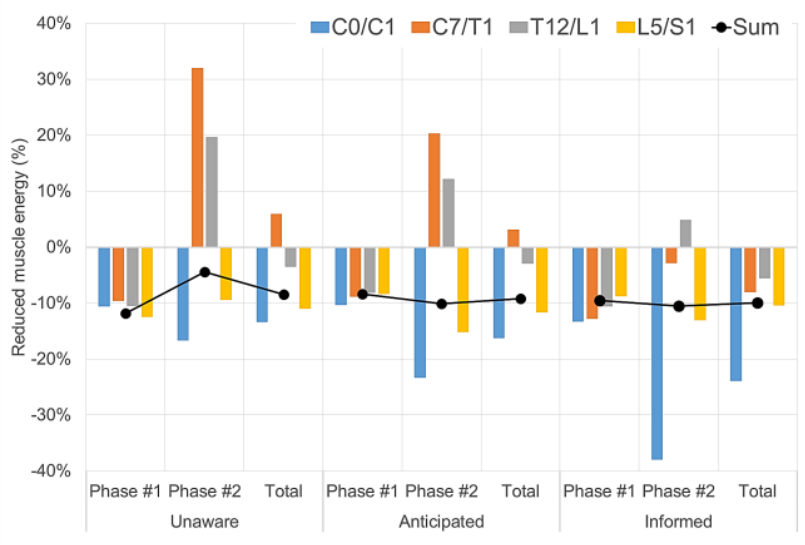

Figure 10. Muscle energy reduction at reduced jerk maneuver

\section{Conclusion}

The active human body model primarily developed for a ride comfort simulation to investigate the vibration response of human occupants is applied to predict relatively short-term ride quality via simulating a kinematics at an evasive lane change. The lateral movements of head and torso at three awareness conditions, unaware, anticipated, and informed were validated against the vehicle-based subject test. The simulation result showed different correlation outcomes between the $1^{\text {st }}$ phase and the $2^{\text {nd }}$ phase of the lane change maneuver, a good (0.94 CORA score) versus a poor (0.46 CORA score). It is speculated that the current error function in the PID closed-loop control, which is the difference of the current inclined angles of each body segment from the initial vertical axis, may not be appropriate for the $2^{\text {nd }}$ phase. Unlike the $1^{\text {st }}$ phase in the lane change maneuver where the head and torso are moving away from the initial upright position, that is to say, outbound direction, the $2^{\text {nd }}$ phase where the head and torso are moving inbound direction may have substantially different reflexive muscle activity. In addition, the lateral G-force direction of the vehicle becomes completely opposite in the $2^{\text {nd }}$ phase. Taking this human physiology into account will improve the simulation result of the $2^{\text {nd }}$ phase and still remains as our future research challenge.

In order to verify a practical application of the active human body model in the design process of the ride quality, an artificial single lane change maneuver with reduced lateral jerk was fabricated and its effect on the occupant kinematics was investigated. The model predicted a joint work, regarded as a muscle energy used to maintain the sitting posture against the lateral G-force with a comparable reduction at the created lane change maneuver and successfully demonstrated its feasibility of serving as an objective design tool for quantifying the ride quality.

\section{Acknowledgements}

The underlying data were generated at the VIRTUAL VEHICLE Research Center in Graz, Austria. VIRTUAL VEHICLE Research Center would like to acknowledge the financial support of the COMET K2 Competence Centers for Excellent Technologies Program of the Austrian Federal Ministries, the Austrian Research Promotion Agency (FFG), the Province of Styria and the Styrian Business Promotion Agency (SFG).

VIRTUAL VEHICLE Research Center would furthermore like to express its thanks to its supporting industrial and scientific project partners, in alphabetical order: Bundesanstalt für Straßenwesen-BASt, Robert Bosch GmbH, DYNAmore GmbH, Daimler AG, BMW Group, TRW Automotive, Toyoda Gosei Europe, Partnership for Dummy Technology and Biomechanics, Volkswagen, Graz University of Technology, Vehicle Safety Institute, Graz University of Technology, Institute of Automotive Engineering, LudwigMaximilians-Universität Munich.

\section{References}

H.Y. Choi, M. Han, A. Hirao and H. Matsuoka, Virtual Occupant Model for Riding Comfort Simulation, 12th International Modelica Conference, Czech Republic, 2017.

H.Y. Choi, M. Han and W. Lee, Active Elbow Joint Model, The First Japanese Modelica Conference, 2016

M. Han and H.Y. Choi, Elbow joint model with active muscle force, Journal of Mechanical Science and Technology 30/12 5847 5853, 2016

P. Huber, M. Christova, G. A. D’Addetta, E. Gallasch, S. Kirschbichler, C. Mayer, A. Prüggler, A. Rieser and W. Sinz, D. Wallner, Muscle Activation Onset Latencies and Amplitudes during Lane Change in a Full Vehicle Test, IRCOBI Conference 2013

A. S. Kilinç and T. Baybura, Determination of Minimum Horizontal Curve Radius Used in the Design of Transportation Structures, Depending on the Limit Value of Comfort Criterion Lateral Jerk, FIG Working Week, Knowing to manage the territory, protect the environment, evaluate the cultural heritage, Rome, Italy, 2012

S. Kirschbichler, P. Huber, A. Prüggler, T. Steidl, W. Sinz, C. Mayer and G. A. D`Addetta, Factors Influencing Occupant Kinematics during Braking and Lane Change Maneuvers in a Passenger Vehicle, IRCOBI Conference 2014 
C. Kleinbach and J. Fehr, Comparison of Muscle Activated HBMs in a Lane Change Manoeuvre, IRCOBI Conference 2017

G. Lauriks, J. Evans, J. Förstberg, M. Balli and I. B. de Angoiti, Uic comfort tests: Investigation of ride comfort and comfort disturbance on transition and circular curves. Technical report, Swedish National Road and Transport Research Institute, 2003.

W. Schofield, Engineering Surveying - Theory and Examination Problems for Students, ButterworthHeinemann, Oxford, New Delhi, 2001

J. Takahashi, M. Yamakado and S. Saito, Evaluation of preview $G$-Vectoring control to decelerate a vehicle prior to entry into a curve, International Journal of Automotive Technology, Vol. 14, No. 6, pp. 921-926, 2013

K. Yamada, H. Motojima, Y. Kitagawa and T. Yasuki, Investigation of relations between occupant kinematics and supporting by the seat in lane change maneuvers, 20165176, JSAE Spring Congress, 2016

AASHTO; A Policy on Geometric Design of Highways and Streets, American Association of State Highway and Transportation Officials, Washington DC, USA. 2001

EuroNCAP, Pedestrian Human Model Certification, Version 1.0 TB 024, 2017

International Organization for Standardization. ISO 2631-1. International standard. ISO, 1997.

www.GHBMC.com 